\title{
Modelo longitudinal de estudo de doenças crônicas - um estudo de 26 pacientes com câncer colorretal (CCR)
}

\author{
Acadêmicos: Elias El-Mafarjeh, Sun Woo Ryoo \\ Orientador: Andrew Grant
}

Introdução: O câncer colorretal (CCR) é responsável por diversas mortes em todo o mundo. Este estudo representa uma tentativa preliminar de modelagem longitudinal do CCR, uma doença crônica. Doença crônica que pode ser vista como uma série de eventos médicos que se correlacionam e, portanto, podem afetar os resultados de previsão de prognostico e tratamento.

Objetivo: O objetivo deste estudo é integrar o princípio de Bayesian network(BN) e biologia em uma abordagem sistémica para detectar possíveis interações entre fatores de risco, prognostico e tratamento do CCR.

Metodologia: Este estudo foi realizado com base em dados de 26 pacientes com câncer colorretal, utilizando o Data warehouse do Hospital em que eles foram tratados e assim foi montada a trajetória longitudinal desses pacientes, desde o diagnostico inicial até um período de cerca de 18 meses. Os dados foram organizados no programa Microsoft Access como um banco de dados local, onde todos os dados foram processados e organizados por paciente. Todos os pacientes tiveram seus nomes trocados por números para proteger a sua privacidade. Sendo o acesso dos dados aprovado pelo Institutional Review Board (IRB). Foram feitas duas abordagens. Uma abordagem foi visualizar a trajetória clínica dos 26 pacientes com CCR e estabelecer um cronograma delineado de seus cuidados utilizando os recursos de gráfico do Microsoft Excel, incluindo a ocorrência de cirurgia, quimioterapia e os níveis de antígeno carcino-embrionário (CEA) como variáveis de interesse. A duração da estadia no hospital, sexo e idade foram incluídos para facilitar a análise qualitativa dos dados. Na outra abordagem, a trajetória clínica dos 26 pacientes foram analisados no software Netimis (www.netimis.co.uk) onde as probidades de cada evento foram calculadas de forma empírica com base nesta coorte. Os pacientes foram diferenciados de acordo com a idade no início do tratamento e sexo, as cirurgias e sessões de quimioterapia foram convertido em variáveis categóricas, usando gráficos de dispersão e uma simulação para 500 pacientes hipotético foi executada para avaliar o modelo Netimista em estudos posteriores.

Resultado: Foi montado um cronograma para os dados de cada paciente $(n=26)$ foi produzido mostrando a progressão do tratamento, diagnósticos, cirurgia, remissão e quimioterapia de cada paciente e foi construído um mapa de Netimis que representa a trajetória clínica dos pacientes, sendo as variáveis incorporadas idade, sexo, níveis de CEA, cirurgias e agentes quimioterápicos.

Discussão e Conclusão: A análise de dados longitudinais e a visualização da trajetória do paciente, exames, tratamentos e resultados é de primordial importância. Isso permite que o especialista critique e analise a trajetória do paciente. Este trabalho analisou dois aspectos complementares da análise de trajetória - a representação com base na linha do tempo (Bui et al., 2007; Hawkes et al., 2010) e a representação com base na sequencia de eventos. As duas abordagens tomadas neste estudo preliminar são boas representações visuais de dados longitudinais e, assim, podem ajudar a melhorar a pesquisa clínica e processos tais como o desenvolvimento da hipótese diagnostica (Chakrabarti et al., 2013). Entretanto mais estudos devem ser desenvolvidos sobre como correlacionar os BN a trajetória de pacientes com doenças crônicas. 